\title{
„DiE SChlüSSE AUS DER ANalogie SIND SEHR UNSICHER.“" \\ DIE OFFENEN ENDEN ANALOGER REDE IN LICHTENBERGS NOTATEN
}

Ein technischer Terminus wie Digital-Analog-Wandler impliziert die Fiktion, dass sich analoge Speicherung und Übertragung unmittelbar an die Wirklichkeit ,ursprünglicher' Signale anschmiegen. Doch auch wenn cin bestimmtes Signal analog ,bleibt", so muss es, um speicher- und übertragbar zu bleiben, Metamorphosen durchmachen. Ein akustisches Signal - eine mechanische Schwingung der Luft - wird durch ein Mikrofon in elektromagnetische Schwingungen verwandelt, von denen es wahlweise in mechanische zurückgewandelt werden kann, um die Luftschwingung auf der Patrize einer Vinyl-Schallplatte speicher- und vervielfältigbar zu machen, oder es wird ein Band analog zu diesen Schwingungen magnetisiert; die Rückverwandlung in Schall erfolgt umgekehrt. Auch ein Verstärker operiert analog, da dieser nur die Amplitude der Schwingung variiert, die Verhältnisse der Frequenzen aber unangetastet bleiben. Es ließen sich hieran viele technikgeschichtliche Fragestellungen anschließen, etwa inwiefern ein mit digitaler Technik opericrendes akustisches Effektgerät wie der Harmonizer, der die Grundschwingung eines akustischen Signals in Halbtonschritten auf- und abwärts führt, nicht insofern ein analoges Ergebnis liefert, als das ursprüngliche Signal in einem gegebenen arithmetisch-harmonischen Verhältnis zum Ausgangssignal steht. Um zumindest die Möglichkeit solcher Fragestellungen adäquat zu formulieren, ist es unerlässlich, zunächst eine historisch-philologische Auseinandersetzung mit den Begriffen analog und Analogie vorzunchmen.

Der altgriechische Begriff analogia, der in der lateinischen Sprache mal treffender mit proportio wiedergegeben, mal mit similitudo am genauesten übersetzt ist, entspricht im Deutschen den Begriffen Entspre- 
chung, Ähnlichkeit, Verhältnis, Vergleich, auch Gleichnis.' Die technische Verwendung des Begriffs, analog' geht auf die von den Vorsokratikern herrührende erste bekannte Bedeutung dieses Terminus zurück, die nicht im rhetorischen, sondern im mathematischen Bereich zu finden ist. Es geht hicrbei, vereinfacht gesagt, um Proportionalität: die Bestimmung von Verhältnissen. Bis in das Mittelalter hincin ist dic Berechnung von Verhältnissen das Hauptanliegen der zahlenmäßigen Disziplinen, die unter dem Dach der septem artes liberales im Quadrivium untergebracht sind; hier finden sich die Wissensdisziplinen, die sich der res, der Dinge der Welt, in mathematischer Abstraktion annehmen. Es sind dies die Arithmetik (Verhältnisse in Zahlenreihungen), die Geometrie (Verhältnisse in Flächen), die Musik (harmonische Verhältnisse von Tönen), die Astronomic (harmonische Verhältnisse der Planetenbahnen). Schon bei der mathematischen Analogie geht es darum, Unbekanntes aus Bekanntem zu schließen. In der Arithmetik etwa lässt sich aus der gegebenen Zahlenreihe 1, 2, 4 unter Eruicrung der Formel $f(n)=2^{n}$ (für $n \geq 0$ ) schließen, dass die folgenden Zahlen 8, 16, 32 etc. lauten müssen. Stimmen bei zwei gegebenen Dreiecken zwei Winkel überein, ist daraus zu schließen, dass diese beiden Dreiecke ähnlich (= ana$\log$ ) sind.

Schon früh wird die Analogie in dic christlich-theologische Argumentation übersetzt, so etwa in der Typologie (harmonisiert dic Aussagen des Alten und Neuen Testaments) oder spezieller in der Analogia Fidei; der entsprechende Eintrag im Zedler betont, dass der Begriff der Analogia „sonst nur bey denen Mathematicis bekant [sei][...]. Aus der Schule derer Mathematicorum nun ist es [die Analogia] in die heilige Disciplinen gebracht worden, wie denn der heilige Apostel Paulus selbst sich dieses Wortes Rom. XII. 6. bedienet."

Der Begriff der Analogia Fidei meint nun - und dies ist, freilich übertragen, auch bei Lichtenberg wichtig -, dass selbst Aussagen, dic sich offensichtlich widersprechen, sich bei genauerer Betrachtung gemäß der Universalität des Glaubens doch wieder entsprechen. Als Beispiel greift der Artikel im Zedler auf dic Rechtfertigungslehre zurück; aus Röm 3,27 wird das bekannte Pauluswort zitiert, dass der Mensch nur durch den Glauben an Jesus Christus, nicht aber durch die Werke ge-

1 Im Folgenden rekurriere ich auf den $\Lambda$ rtikel Analogie von Hoenen, in: Gert Ueding (Hrsg.): Historisches Wörterbuch der Rhetorik, Tübingen 1992ff., Bd. 1, Sp. 498-514.

2 Zedler, Johann Heinrich: Grosses vollständiges Universal-Lexicon Aller Wissenschaften und Künste [fortan als Zedler], Leipzig/Halle 1732-54, Repr., Graz 1961 ff., Bd. 2, Sp. 34. 
rechtfertigt wird, in Jak 2,24 hingegen steht, dass man auch durch dic Werke, nicht aber durch den Glauben allein gerecht werde. Ein Widerspruch?

Ach nein, keinesweges, denn wenn wir erwegen, daß Paulus von der Gerechtfertigung für GOtt, und hingegen Jacohus von der Gerechfertigung für denen Menschen, Paulus mit annoch Unwiedergebohrnen, Jacobus aber mit Wiedergebohrnen handele, so werden wir gantz leicht erkennen, daß sie vollkommen einig sind, und daß keiner $\mathrm{dem}$ andern widerspreche, und so ist es mit denen andern Stellen mehr. ${ }^{3}$

Von großer Bedeutung ist die Analogie freilich für den Bereich der Rhetorik. Der theoretische Witz der rhetorischen Analogie besteht darin, dass sie genau genommen selbst eine rhetorische Analogic ist, nämlich cine der mathematischen Analogie - auch hier geht es um Verhältnisse, wenn gleich übertragener Art; Aristoteles definiert:

Unter einer $\Lambda$ nalogie verstehe ich eine Beziehung, in der sich die zweite Größe zur ersten ähnlich verhält wic die vierte zur dritten. Dann verwendet der Dichter statt der zweiten Größe die vierte oder statt der vierten die zweite [...]: Das $\Lambda$ Iter verhält sich zum Leben, wie der $\Lambda$ bend zum Tag; der Dichter nennt also den $\Lambda$ bend , $\Lambda$ lter des Tages", oder, wie Empedokles, das $\Lambda$ iter,$\Lambda$ bend des Lebens* oder ,Sonnenuntergang des Lebens'. ${ }^{4}$

Innerhalb der Rhetorik ist die Analogic Grundlage von Vergleich, Metapher, Allegoric und Emblem, also für das Bearbeitungsstadium der elocutio, in dem die Rede mit Tropen und Figuren ausgestattet wird; aber auch für die Bearbeitungsstufe der inventio, der Stofffindung, ist sie von Bedeutung wie auch für den Redeteil der argumentatio, der Beweisführung. Dies ist für unseren Kontext wichtig, da die Analogie hier wesentlich zur persuasiven Funktion der Rede beiträgt, sei es als Beweis eines Sachverhaltes, sei es in seiner didaktischen Vermittlung in der similitudo, dem Gleichnis. Im Vergleich zu ihrer Situierung in der Mathematik ist dic Analogic in der Rhetorik freilich unberechenbar, wodurch dieser einerseits die Beschränktheit, andererseits die implizite Evidenz mathematischer Sätze abgeht.

Wie man aus den skizzierten Eckdaten des Analogiebegriffs leicht cinsehen kann, ist eine Begriffs-, Wort- oder Metapherngeschichte der Analogie kaum in einem kleinen Aufsatz abzuhandeln. Daher ist es sinnvoll, die Texte eines Autors zu lesen, der am Schnittpunkt verschiedener

3 Zedler $(\Lambda \mathrm{nm} .2)$, Sp. 34f.

4 Aristoteles: Poetik, hrsg. v. Manfred Fuhrmann, Stuttgart 1982, 1457b. 
Textgattungen und Wissensfelder schreibt und dabei eine Situierung der Analogie betreibt, die deren Problematik, aber auch ihre Potenzialität belcuchtet.

Georg Christoph Lichtenberg (1742-1799) war einer der besten Mathematiker und Experimentalphilosophen seiner Zeit; cr wurde 1775 Mitglied der Royal Society of London und drei Jahre später der Akademic von St. Petersburg. Scine Antrittsvorlesung zur Wahrscheinlichkeitstheoric und seine elektrostatischen Experimente, ${ }^{5}$ die zur Entdeckung der Lichtenberg-Figuren führten, stellen nur einen kleinen Ausschnitt seiner Forschungsbereiche dar.

Zwar hat Lichtenberg zeit scines Lebens umfassend Aufsätze publiziert - vornehmlich im Göttinger Taschen Calender und im Göttingischen Magazin der Wissenschaften und Litteratur, die cinzige größere Publikation zu Lebzeiten ist Erklärungen der Hogarthischen Kupferstiche (1794-99) - doch der literarische Nachruhm ist in erster Linie seinen postum edierten Sudelbïchern zu verdanken, die nach 1770 bis zu seinem Tod entstanden. Mit ihnen hat er eine Textgattung geschaffen, die, da sie weder Systematik noch geschlossene argumentative Ordnung kennt, nicht der Philosophie angehört, auf Grund der Weite der Themenbereiche aber auch keineswegs der Belletristik zugeschlagen werden kann. Die Einträge in den Sudelhüchern bestehen aus Notizen, Bemerkungen, skizzierten Gedanken(fragmenten), Fragestellungen zu fast allen Themen und Wissensbereichen, von Rhetorik und Stilistik über das gesellschaftliche Leben hin zu Mathematik und Naturphilosophic; auch die Auscinandersetzung mit der Physiognomik Lavaters in seiner Streitschrift (Üher Physiognomik; wider die Physiognomen (1778)) findet hier ihren Ausgangspunkt. Die Germanistik hat sich eine Zeit lang damit zufrieden gegeben, die Notate Lichtenbergs unter dem Rubrum der Aphoristik einzuordnen, doch erwies sich diese Kategorisierung zunchmend als problematisch.

Besonders für Lichtenberg als Naturphilosophen ist die Analogie von Bedeutung, indem sic ,Neues' begreifbar, beschreibbar, benennbar macht, und zwar qua Rückanbindung an je bestehende Wissensformationen. Die Analogie, ob dies nun in den jeweiligen Applikationen deutlich wird oder nicht, spielt in ihrer Brückenfunktion dabei sozusagen eine Doppelrolle: Indem sie Altes in Neuem erkennbar macht, stellt sie Kontinuität und Differenz her.

5 Auf Lichtenberg gehen auch die Bezeichnungen, ,+' und, ' für positive und negative Ladungen zurück. 
Bereits in einem frühen Stück aus dem ersten Sudelbuch von 1770 formuliert Lichtenberg: „Die Natur schafft keine genera und keine species, sie schafft individua und unsere Kurzsichtigkeit muß sich Ähnlichkeiten aufsuchen um vieles auf einmal behalten zu können." bringt Lichtenberg noch einmal zum Ausdruck, was bereits in den skeptizistischen Vorgaben empirischer Naturphilosophie - bei Autoren wie Glanvill, Boyle und Newton - ausdrücklich formuliert wurde, nämlich, dass die Erkenntnisfähigkeit des menschlichen Verstandes minim ist und die wahre Natur der Natur niemals erkannt werden kann. Seit Lockes Essay Concerning Human Understanding und vor allem seit dessen Rezeption in Addisons Essays on the Pleasures of the Imagination wird diese Defizienz des menschlichen Verstandes umgewertet bzw. erkenntnistheoretisch kompensiert durch die Fakultät der Einbildungskraft, die im 17. Jahrhundert noch grosso modo als Teufelswerk galt, wenn die Vernunft ihr nicht die engsten Grenzen setzte. Die Einbildungskraft wird zunchmend als Instrument zur Erlangung von Erkenntnissen cingeschätzt, dessen sensuelle Vorgaben freilich noch aus der materiellen Natur kommen, die aber derart umgestaltet und bearbeitet werden, dass der Verstandestätigkeit zunchmend eine relative Autonomic und Individualität zugeschrieben wird; diese ist von den konkreten Vorgaben der materiellen Natur weitgehend unabhängig. Somit ist cine Entwicklung bereits um 1700 vorgegeben, die bei Hume und Kant endet.

Für die Begriffe species, genera, individua nennt Gockel nun als einzige Referenz Aristoteles' Organon, ${ }^{7}$ doch ist zu bedenken, dass diese Begriffe auch für die Taxonomic frühneuzeitlicher Wissenschaft valent sind. Somit rekurriert Lichtenberg auch auf den Radikalskeptizismus der empirischen Naturphilosophic angelsächsischer Prägung, wenn cr genera und sogar species schlichtweg als nicht existent bezeichnet und nur die individua gelten lässt und somit den Wert der ordnenden Arbeit der Taxonomen des 17. und 18. Jahrhunderts - Ray, Linné und Buffon - zumindest einschränkt. Um dennoch etwas über die Natur sagen und dieses Gesagte im Gedächtnis festschreiben zu können, muss sich unsere ,Kurz-

6 A17. Ich zitiere hier und im Folgenden nach der Ausgabe: Lichtenberg, Georg Christoph: Schriften und Briefe, hrsg. v. Wolfgang Promies, München $1968 \mathrm{ff}$. Wie in der Lichtenbergforschung üblich und zum Nutzen und Frommen des Lesers gebe ich immer die Sudelbuch-Sigel und die jeweilige fortlaufende Fragmentnummer an; zur besseren Orientierung versehe ich die Angaben, die sich auf den zweiten Band der Ausgabe bezichen mit ${ }_{\text {II }}$.

7 Vgl. Gockel, Heinz: Individualisiertes Sprechen. Lichtenhergs Bemerkungen im Zusammenhang von Erkenntnistheorie und Sprachkritik, Berlin/New York 1973, S. 21. 
sichtigkeit` Ähnlichkeiten zwischen konkreten individua suchen. Bemerkenswert ist hieran zweierlei: Zum einen deutet schon dieses frühe Fragment auf die bei Lichtenberg erst später bemühte (und hier weiter unten besprochene) optische Metaphorik innerhalb sciner Erkenntnistheorie, auf der anderen Seite verficht Lichtenberg ausdrücklich eine individualistische und anthropomorphistische Haltung in Hinsicht auf Wahrnehmung, Denken, Sprache und Stil, die mit ihrer generellen Defizienz hinsichtlich der Erfassbarkeit von Natur rechnet. In einem späteren Stück, das sich auch mit den Begriffen von genera und species beschäftigt, führt er, schon deutlich unter dem Einfluss Kants stehend, aus: „Überhaupt kann man nicht gnug bedenken, daß wir immer nur uns beobachten, wenn wir die Natur und zumal unsere Ordnungen beobachten. ““ Dabei ist die Analogie - hier nicht im Sinne von , Ähnlichkeit‘, sondern von ,Verhältnis' - bereits durch die Einrichtung unserer Sinne vorgegeben: „Unsere Sinnen haben eine solche Verhältnis zu den Dingen um uns, daß bei ihrer Entstehung schon das Wichtigste geschehen ist, ehe wir wissen, daß sie da sind [...]." An dieser Stelle wird eine Grunddifferenz der Sinne und der materiellen Welt beschrieben, die allerdings konstitutiv für Wirklichkeitserfahrung überhaupt ist.

Es kommt hinsichtlich des Begriffs der Analogie nun eine gewisse Schwicrigkeit hinzu: Gemeinhin kommt dic Analogic zum Einsatz, wenn Unbekanntes bekannt gemacht werden soll, indem zwischen dem Bekannten und dem Unbekannten eine Ähnlichkeit hergestellt wird; unterstellt ist somit cine prinzipielle Diskontinuität, dic durch dic Analogic mehr oder weniger , vorübergehend " - unterbrochen wird. ${ }^{10}$ Bei Lichten-

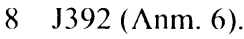

$9 \Lambda_{11} 223(\Lambda \mathrm{nm}$. 6), vgl. auch D212 $(\Lambda \mathrm{nm}$. 6), wo Lichtenberg auf die Ähnlichkeit der beiden Bilder eingeht, die unsere beiden $\Lambda$ ugen liefern: Es dünke ihn „,daß wir alle Sachen zweimal sehen, aber ohne allen Unterschied wegen der ähnlichen Lage der Bilder gegen unsern symmetrischen Körper, und daher sie für eins halten. [...] Die Verhältnis der Gleichheit (könnte man oben beim Auge sagen) ist vielleicht in der Seele $=0$ so wie es in der $\Lambda$ rithmetik bei der Zusammensetzung der Verhältnisse ist." $\Lambda$ n dieser Stelle steht die ganze ,Simplizität der Seele" auf dem Spiel; die Seele wird zum Ort eines generellen ,doppelten Blicks', der zu sich selbst in einem analogen Verhältnis steht; letztendlich ist die Seele mit diesem Verhältnis identisch. Diese Konstellation und ihre immensen Konsequenzen hier eingehend und angemessen zu diskutieren, würde den Rahmen dieses Textes sprengen.

10 Über diese Situierung der $\Lambda$ nalogie ist sich Lichtenberg völlig im Klaren, vgl. etwa L665 ( $\Lambda \mathrm{nm}$. 6), wo er von „Vergleichungen zwischen sehr heterogenen Dingen" spricht; vgl. auch $J_{11} 1446(\Lambda \mathrm{nm} .6)$, „Zu der Zeit, da man sich mit einer Sache beschäftigt, da sie einem völlig geläufig und gegen- 
berg ist nun der Witz, dass zwischen den Dingen der materiellen Natur die Differenz nur gradueller und nicht prinzipieller Natur ist:

Mir scheint überhaupt in der Natur alles durch Verhältnisse in den Ursachen, nicht durch wesentliche Verschiedenheit bewirkt zu werden. Dieses ist wieder eine $\Lambda$ nwendung von meinem Alles in allem. Die Menschen sind sich in ihren $\Lambda$ nlagen alle gleich, nur die Verhältnisse machen den Unterschied; und nur die verschiedenen Verhältnisse machen den Mann und den Spitzbuben. ${ }^{11}$

So differenziert Lichtenberg zwischen den Verhältnissen der Dinge untereinander einerseits und denen, die die Dinge und die Menschensinne miteinander unterhalten andererseits. So schreibt er in einem Stück zu seiner, Philosophic' des Alles in allem:

Ich habe schon einmal an einem andern Ort bemerkt, daß sich alles in der Welt in alles verliert, und alles in allem befindlich ist, ich meine alles, was wir bemerken und mit einem Wort bezeichnen, war schon da ehe es zu dem Grad kam, den wir bemerken. Das Beispiel vom Gewitter, daß jede Wolke eins ist, und daß sic bloß dem Grade nach verschieden sein kann. Nichts leitet leichter auf den Gedanken. Vielleicht sind immer Nordscheine und wir nennen nur hohe Grade so, die uns in die Sinne fallen, so könnte jemand geschlossen haben, der nicht wüßte, daß sie fast beständig in den nördlichen Gegenden sind. ${ }^{12}$

In einem anderen heißt es: „Die Metapher ist weit klüger als ihr Verfasser und so sind es viele Dinge. Alles hat seine Tiefen. Wer Augen hat, der sicht [alles] in allem." ${ }^{13}$

In dem ersten Stück behandelt Lichtenberg die Natur, an sich", wie sic sich darstellt, bevor der Mensch sic erblickt und sich Worte und Meinungen dazu bildet. In dieser Idee von der Natur steht alles mit allem in einer Verbindung, die durch die bloße Analogie gar nicht hinreichend gefasst werden kann. Lichtenberg betont, dass die Dinge sich in einem kontinuierlichen, pragmatischen, ,natürlichen' Zusammenhang befinden; diesem entspricht in rhetorischen Termini aber die Metonymie, die sozu-

wärtig nach allen ihren Teilen ist, mu/.3 man suchen sie allem anzupassen auch often den entferntesten Gegenständen, durch Gleichnisse, Analogien erläutern und andere Sachen mit ihr."

$11 \mathrm{~L}_{11} 916(\Lambda \mathrm{nm} .6)$; zu Lichtenbergs Alles in allem vgl. F48, 147, 369, 694, $\mathrm{L}_{11} 915$ ( $\left.\Lambda \mathrm{nm} .6\right)$ sowic Lichtenberg: Schriften und Briefe $(\Lambda \mathrm{nm} .6)$, Bd. 3, S. 265 und 290, vgl. auch B101( $\Lambda$ nm. 6): „Er war so witzig, daß jedes Ding ihm gut genug war zu einem Mittelbegriff jedes Paar andere Dinge mit einander zu vergleichen."

12 F147 ( 1 nm. 6).

13 F369 (Anm. 6). 
sagen das ,Gegenteil' der - auf der Analogie basierenden - Metapher ist, da sie nicht eine Übertragung von einem Sinnbereich in einen anderen ist, sondern ihre Tropen dem gleichen Sinnbereich entnehmen muss. Damit verfügt sie freilich nicht über das Ausdruckspotenzial der Metapher, konstituiert aber einen Realbezug zwischen eigentlichem und übertragenem Ausdruck, der der Metonymic eine größere Evidenz verleiht. ${ }^{14}$ In dem zweiten Stück aber bringt er die apperzeptive Leistung des Menschen ausdrücklich mit der Metapher, also der Rhetorik der Analogie, zusammen. Es ist, als ob die Metaphernbildung des Menschen die ,metonymische“ Strukturierung der Natur simulieren sollte, da diese generell unnachvollzichbar ist. Die Findung einer Ähnlichkeit ist bei Lichtenberg die Sache der Denkungsart des, Witzes“, die auf den englischen Begriff wit verweist und eine weit ausschweifende, rege Geistestätigkeit meint, die imstande ist, sehr heterogene gedankliche Felder zu integrieren. ${ }^{15}$ Dieser gedanklich-sprachliche Witz hat für Lichtenberg cine immense anthropologische Dimension; nicht nur, weil er ein Spezifikum menschlichen Wissenserwerbs ist, sondern das ist, was den Menschen erst zum Menschen macht: „Ohne Witz wäre eigentlich der Mensch gar nichts, denn Ähnlichkeit in den Umständen ist ja alles was uns zur wissenschaftlichen Erkenntnis bringt, wir können ja bloß nach Ähnlichkeiten ordnen und behalten. Die Ähnlichkeiten liegen nicht in Dingen, vor Gott gibt es keine Ähnlichkeiten."16

Lichtenberg ist sich über die abendländische Situierung der Analogic - mathematische Gewissheit vs. rhetorische Offenheit bzw. Vagheit - ganz im Klaren und re-konstituicrt dic Analogic als Didaxe und Erkenntnisinstrument, das es crlaubt, von Bekanntem auf Unbekanntes zu schließen. So weist er ausdrücklich auf die mathematische Bestimmung von Verhältnissen und deren Grenzen hin, wenn er schreibt: „Es gibt viele Verhältnisse in der Natur, die nicht arithmetische und nicht geometrische sind, und die uns daher schwer werden anzugeben [...]."

$14 \mathrm{Vgl}$. Ueding, Gert/Bernd Steinbrink, Bernd: Grundri/3 der Rhetorik. Geschichte, Technik, Methode, Stuttgart/Weimar ${ }^{3} 1994$, S. 294.

$15 \mathrm{Vgl}$. die konzise Darlegung des Witz-Begriffs bei (jockel: Individualisiertes Sprechen $(\Lambda \mathrm{nm} .7)$, S. 75-91, siche auch unten die optische $\Lambda$ llegorisierung des Witzes.

$16 \mathrm{~J} 959(\Lambda \mathrm{nm} .6)$.

$17 \Lambda_{\mathrm{Il}} 156(\Lambda \mathrm{nm} .6)$, vgl. auch die arithmetische $\Lambda$ pplikation des Begriffs , Verhältnis“ in $\Lambda_{\mathrm{II}} 261$ ( $\Lambda \mathrm{nm}$. 6). Lichtenberg, der die Erfindung von $\Lambda$ nalogien als wesentliche Eigenschaft des Witzes definiert (s.u.) rekurriert auch auf die Musik als Berechnung von Verhältnissen: „Es ist mit dem Witz wie mit der Musik, je mehr man hört, desto feinere Verhältnisse verlangt man." 
Da Lichtenberg auch in der empirischen wie skeptizistischen Tradition angelsächsischer (und mithin protestantischer) Naturphilosophie stand, dürfte ihm diese Einschätzung der Nicht-Universalität der Mathematik nicht schwer gefallen sein. Trotz der teils harten Polemik, die die empirische Naturphilosophie des 17. Jahrhunderts der Mathematik zuteil werden ließ, galt sie durchgehend als System in se. Ausgeschlossen aber werden musste, dass die Phänomene der Welt auf mathematische Formeln rückführbar gemacht werden konnten, wie dies grosso modo in den scholastisch geprägten Traditionen der katholischen Ländern der Fall war. $^{18}$ Damit wurde aber auch der Anspruch auf gesichertes Wissen durch quasi-axiomatische Theoreme in der Experimentalphilosophic hinfällig. Dem trägt auch dic Analogic Rechnung, indem Lichtenberg ihr neben ihrer Potenzialität eine gewisse Unsicherheit unterstellt; wer sich der Analogie bedient, setzt sich immer einem Risiko aus: „Die Schlüsse aus der Analogie sind sehr unsicher. Das Viscum quercinum (EichenMistel) wächst nach allen Richtungen unter sich und über sich, da fast alle Pflanzen nur über sich wachsen." ${ }^{19}$ Hierbei referiert Lichtenberg auf einen fast topischen Vorbehalt gegenüber der Analogie; im Zedler etwa heißt es im Eintrag zur Analogia, diese sei „offtmahls ein Deckmantel der Unwissenheit“: „Es ist also keinem zu trauen, der sich nur überhaupt auf die Analogie beziehet, sondern es müssen die gleichen oder die wid-

(D223) Vgl. auch $\Lambda 15(\Lambda \mathrm{nm} .6)$ : ,So wie das Ohr Verhältnisse mißt, so berechnet vielleicht die Zunge Flächen von Körpern."

18 In seinem Aufsatz über dic Wahrscheinlichkeitsrechnung kritisiert Lichtenberg ausdrücklich dic Auffassung, dass Mathematik und empirische Wirklichkeit miteinander zu verrechnen seien: „Der Meßkünstler findet nicht selten bei der Anwendung seiner Schlüsse auf die Natur, merkliche $\Lambda \mathrm{b}$ weichungen von dem, was er nach seiner Rechnung hätte erwarten sollen. Es ist nicht sehr schwer den Grund hiervon im allgemeinen anzugeben, und cinzusehen, daß es nicht die Schuld der Mathematik sein kann" (Lichtenberg: Schriften und Briefe ( $\Lambda \mathrm{nm} .6)$, Bd. 3, S. 9). Zur Problematik der Mathematik vgl. auch Arntzen, Helmut: „Beobachtung, Metaphorik, Bildlichkeit", in: Deutsche Vierteljahresschrift fïr Literaturwissenschaft und Geistesgeschichte, Jg. 42, Nr. 3 (1968) S. 359-372, hicr S. 365.

$19 \mathrm{~K} \Lambda_{\mathrm{II}} 17(\Lambda \mathrm{nm} .6)$. Dass die Unsicherheit der $\Lambda$ nalogie nur eine auf den Punkt gebrachte Konkretisierung der Unsicherheit der „Wörter-Welt" (J359) überhaupt ist, sei hier nur am Rande erwähnt; vgl. hierzu den wenn auch im Titel etwas überzogenen $\Lambda$ ufsatz von McCarthy, John A.: „Lichtenberg as Poststructuralist", in: Charlotte M. Craig (Hrsg.): Lichtenherg. Essay Commemorating the 250 ${ }^{\text {th }}$ Anniversary of his Birth, New York u.a. 1992, S. 1-10, hier S. 2f., sowie Patzig, Günther: „Über den Philosophen Lichtenberg“, in: Text + Kritik, Heft 114 (1992) S. 23-26. 
rigen Sätze ins besondere angeführt werden, daß man daraus die Gleichheit oder Ungleichheit erkennen könne. “20

Die Analogie steht also immer unter dem Vorbehalt, falsch zu sein; wann aber eine Analogie ,richtig' und treffend ist, dafür benennt Lichtenberg keine generelle Grundregel. Die Richtigkeit der Analogie kann sich eben immer nur am jeweiligen individuellen Einzelfall erweisen. Gleichwohl versucht Lichtenberg, ein Bild der richtigen Analogiefindung zu geben:

Wenn man einen guten Gedanken liest, so kann man probieren, ob sich etwas $\ddot{\Lambda}$ hnliches bei einer anderen Materie denken und sagen lasse. Man nimmt hier gleichsam an, daß in der andern Materie etwas enthalten sei, daß diesem ähnlich sei. Dieses ist eine $\Lambda \mathrm{rt}$ von $\Lambda$ nalysis der Gedanken, die vielleicht mancher Gelehrter braucht ohne es zu sagen. ${ }^{21}$

Dic Metapher der Probe, die dic Echtheitsprobe ciner Münze, aber auch den (al)chemischen Versuch konnotiert, kann aber auch nicht als allgemeine Anweisung dienen, sondern ist ihrerseits als eine Analogie des Vorgangs der Analogiefindung zu werten. Insofern ist die Sache der Lichtenberg'schen Analogie tautologisch, da sie nur über eine konkrete Analogie dargestellt werden kann. Dabei wird aber gerade dic prinzipielle Offenheit der Analogie nicht getilgt, sondern bleibt als Potenzial erhalten, freilich nur um den Preis, dass die ,Unsicherheit" und auch eine gewisse ,Einmaligkeit" der Analogic in Kauf genommen werden muss, die unter Umständen gar nicht mehr vermittelbar ist: „Relationen und Ähnlichkeiten zwischen Dingen zu finden, die sonst niemand sicht. Auf diese Weise kann Witz zu Erfindungen leiten.“22 Ein möglicher ,Probierstein' ist die nachträgliche Arbeit des Verstandes, der die gefundenen Ähnlichkeiten prüft: „Der Witz hascht näher oder ferner vom Ende ciner Ähnlichkeit, und der Verstand prüft sic und findet sic richtig, das ist Erfindung." Doch auch diese Beschreibung steht unter einem gewissen Vorbehalt, denn: „So war Isaac Newton.“" ${ }^{23}$ Ein Vorbehalt ist dies deshalb, weil Newton bis weit ins 18. Jahrhundert hinein schon topisch als das naturwissenschaftliche Genie galt, dessen Verstandesfähigkeit die seiner Mitmenschen weit hinter sich ließe. ${ }^{24}$ Zwar kann auch der gemeine

20 Zedler (Anm. 2), Bd. 2, Sp. 35.

$21 \Lambda 76(\Lambda \mathrm{nm} .6)$.

$22 \mathrm{H}_{11} 86($ ( nnm. 6).

$23 \mathrm{~J} 1195$ (Anm. 6).

24 Die Panegyrik Newtons gipfelt in Popes Epitaph: „Nature, and Nature's Laws lay hid in Night. I God said, Let Newton be! and All was Light." 
Naturphilosoph mit Hilfe seines Verstandes seine Erkenntnisse qua Analogic absichern, doch dic Evidenz, die durch die Verstandeskräfte Newtons zugesichert wird, war für sie schlichtweg nicht zu haben. ${ }^{25}$

Für sich selbst stellt Lichtenberg - in einer weiteren Tilgung jeglicher Unsicherheit - klar: ,Wenn ich irgend in etwas Stärke besitze so ist es gewiß im Ausfinden von Ähnlichkeiten und dadurch im DeutlichMachen dessen was ich vollkommen verstehe, hierauf muß ich also vorzüglich denken."26 Er greift hierbei deutlich auf einen Ton zurück, der dem illokutiven Sprechakt eines Befehls nahekommt. Der Befehl duldet keinen Widerspruch und er erhebt die Findung der richtigen Analogie zum kategorischen Imperativ des Schriftstellers: „Ein guter Schriftsteller hat nicht allein Witz nötig, dic Ähnlichkeiten auszufinden, wodurch er seinem Ausdruck Anmut verschaffen kann, sondern auch die zu vermeiden, die dem Leser zum gänzlichen Verderben desselben einfallen können." 27

Die Absicherung, Findung und Ausarbeitung einer richtigen Analogie wird von Lichtenberg enggeführt mit dem Bereich der Moralität. Die richtige Analogie soll die Moralität der Leserschaft befördern und steht somit selbst unter dem Verdikt und der Kontrolle der Moralität, womit sie abermals Offenheit aufgibt, um diesmal auf der moralischen Ebene abgesichert zu scin.

(Pope, Alexander: Minor Poems, hrsg. v. Norman Ault/John Butt, London/New York 1993, S. 317).

$25 \mathrm{Vgl}$. hierzu auch Arntzen: „Beobachtung, Metaphorik, Bildlichkeit“ ( $\mathrm{nm}$. 18), S. 362, der das Problem tilgt, indem er dem individuellen Blick auf die Dinge die Gewähr, richtiger' Bildlichkeit zuschreibt; Arntzen verrechnet diese Individualität aber vorschnell mit neuzeitlicher Subjektivität: ,nur ein Subjektives, das nie dogmatisch, immer kritisch ist, verbürgt Objektivität." Dabei übersicht er, vielleicht beflügelt vom Erscheinungsjahr seines Aufsatzes, dass diese Konstellation des, individuellen Blicks" ihre Valenz erst durch die quasi-juridischen, empirisch-experimentellen Prozeduren der naturphilosophischen Sozietäten Europas erhält.

$26 \mathrm{~J}_{\mathrm{II}} 1646(\Lambda \mathrm{nm} .6)$.

$27 \mathrm{H}_{\mathrm{II}} 72(\Lambda \mathrm{nm} .6)$. $\Lambda \mathrm{n}$ dieser Stelle kann ich nur kurz bemerken, dass es eine $\Lambda$ rt der Analogiefindung gibt, gegen die sich Lichtenberg zeit seines Lebens heftig verwehrt hat: nämlich die Physiognomik Lavater'scher Prägung, die Analogien zwischen dem äußeren Erscheinungsbild eines Menschen und dessen Moralität und geistigen Fähigkeiten konstruiert. Als Gegenkonzept spielte Lichtenberg mit dem (unausgeführten und tatsächlich unausführbaren) Gedanken ciner "Physiognomik des Stils“ (F802 ( $\Lambda \mathrm{nm} .6)$, vgl. hierzu auch Schönc, Albrecht: Aufklärung aus dem Geist der Experimentalphysik. Lichtenhergsche Konjunktive, München ${ }^{3}$ 1993, S. 7-14). 
Um aber doch noch die Ungewissheiten der Analogic mit der Evidenz der Mathematik zu imprägnieren, greift Lichtenberg auf die Metaphorik der Dioptrik zurück. Darin folgt er Robert Boyle, einem der bedeutendsten Naturphilosophen der zweiten Hälfte des 17. Jahrhunderts und Mitgründer der Royal Society; dieser benannte Metaphern und Analogien als wichtige didaktische Hilfen für die Vermittlung naturphilosophischer Sachverhalte. In einem Text aus den 80er Jahren des 17. Jahrhunderts - in dem die Mikroskopic aus verschiedenen Gründen ihre wissenschaftliche Valenz verlor $^{28}$ - taugt diesem das Mikroskop gewissermaßen als die Analogie der Analogic. Dabei figuriert das Mikroskop nicht in erster Linie als, Inhalt' des Textes, sondern als Bild von Übertragung und Übermittlung schlechthin. Bereits 1690 legitimierte Boyle in The Christian Virtuoso den Gebrauch von "metaphors, allegories, and other resemblances", wenn diese Vergleichstropen ,proper" gewählt und der perspicuitas - der rhetorischen ,Durchsichtigkeit" - einer zu erläuternden Sache dienlich seien, ,[t]o which I shall add, that proper comparisons do the imagination almost as much service as microscopes do the eye [...]. “29

Interessant ist hieran, dass die zwei abendländischen Analogietraditionen an dieser Stelle enggeführt werden. Der Strahlensatz, nach dem die analoge Vergrößerung oder Verkleinerung eines geometrischen Gebildes zumindest theoretisch bewältigt werden kann, findet seinen materiellen Niederschlag (idealiter!) ${ }^{30}$ in den Lichtbrechungen des Mikroskops oder Teleskops: „Das Mikroskop vergrößert, die Flächen (Linien), läßt dic Winkel ungeändert, und verkleinert dic Krümmungen." ${ }^{31}$ Die rhetorische Analogie borgt sozusagen ihre Evidenz von

28 Warum das so war, vgl. den $\Lambda$ ufsatz von: Verf./Witthaus, Jan-Henrik: „(jenauigkeit und Vorstellung. Optik, Imagination und die Rhetorik der evidentia im 17. und frühen 18. Jahrhundert", in: Scientia Poetica, Jg. 7 (2003) S. 49-82.

29 Boyle, Robert: The Works [1772], hrsg. v. Thomas Birch, Hildesheim 1965, Bd. 5, S. $511 \mathrm{f}$; das Zitat lautet weiter: ,[...] for, as this instrument gives us a distinct view of diverse minute things, which our naked eyes cannot well discern, because these glasses represent them far more large, than by the bare eye we judge them; so a skillfully chosen and well applied comparison much helps the imagination, by illustrating things scarce discernible, so as to represent them by things much more familiar and easy to be apprehended."

$30 \mathrm{Zu}$ dem Problemen, mit denen die Dioptrik, speziell in Bezug auf das Mikroskop, zu kämpfen hatte, vgl. Hacking, lan: „Do We See Through a Microscope?", in: Pacific Philosophical Quarterly, Jg. 63 (1981) S. $305-$ 322, hier S. 310f. u. passim.

$31 \Lambda_{\text {II }} 187($ (nm. 6). 
der mathematisch-optischer Gleichungen, die in dioptrischen Medien ,realisiert' werden.

Eine ähnliche Konstellation findet sich bei Lichtenberg, nur dreht er das Beispiel Boyles um, indem er das Verkleinerungsglas als Metapher des ,Witzes“ liest: „Scharfsinn ist ein Vergrößerungs-Glas, Witz cin Verkleinerungs-Glas. Das letztere leitet doch auf das Allgemeine. “32

So hat man also den sezierenden, in die Feinstruktur der Dinge eindringenden Blick des (metonymisch für Mikroskop und Teleskop stehenden) Vergrößerungsglases. Gegen diesen Blick wurde von Autoren wie Locke und Addison polemisiert, da er im Ruch stand, den Naturforscher von der menschlichen Umgebung und den anderen Weltdingen abzuschotten, ${ }^{33}$ Lichtenberg trägt diese Polemik hier nicht mit, will den mikroskopischen Blick aber ergänzt sehen durch das Verkleinerungsglas. Warum? Mit dem Fernglas und dem Mikroskop wird ein ganz bestimmter minimer Ausschnitt vergrößert, mit dem Verkleinerungsglas bekommt man ein Sichtfeld geboten, das komprimiert auf den kleinsten Raum die unterschiedlichsten Gegenstände zeigen kann. Unter Umständen kann man so, etwa bei der Betrachtung von Landschaften, Objekte in dieser auf einen Blick sehen, für deren Erfassung man ansonsten den Blick schweifen lassen müsste. Wie in der Analogie wird Fernliegendes zueinander in Bezichung gesetzt.

Lichtenberg gibt zwei konkrete Anwendungsbeispiele, ${ }^{34}$ bei denen die Verkleinerung einen Mehrwert gegenüber der Vergrößerung hat; zum einen hinsichtlich der Einfachheit der Darstellung, zum anderen hinsichtlich der Vorstellbarkeit von Naturvorgängen. „Beobachteten wir den Mond mit umgekehrten Fernröhren, und machten aus dem Okular-Glas das Objektiv-Glas, so würden die Tafeln weit einfacher werden können,

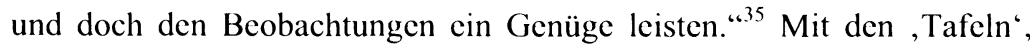
die von Halley, Euler und Mayer herausgegeben wurden, sind Mondsta-

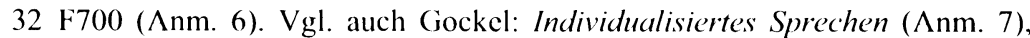
S. 82. $\mathrm{Zu}$ unterscheiden ist der hier zitierte Gebrauch von dioptrischen Medien als Metapher von der Situierung dioptrischer Medien als Grundlage von $\Lambda$ nalogien, wie etwa in F559 ( $\Lambda \mathrm{nm} .6)$ : „Auch ist Mikroskop und Verkleinerung-Glas, mit analogischen Schlüssen verbunden, ein Haupt-Mittel zur Erfindung."

33 Vgl. hierzu Verf./Witthaus: „Genauigkeit und Vorstellung“ ( $\Lambda \mathrm{nm} .28)$.

34 Verkleinerungs- und Vergrößerungsfantasien finden sich z.B. $\mathrm{J}_{11} 1488$ und 1645 (Anm. 6); in E368 beschreibt er Saturn und seine Monde als ein verkleinertes ,Modell` des Sonnensystems. Vgl. hierzu Blumenberg, Hans: Die Genesis der kopernikanischen Welt, Frankfurt a.M. 1969, S. 687 und 783f. sowie ders.: Die Lesharkeit der Welt, Frankfurt a.M. 1999, S. 210.

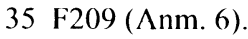


feln gemeint, „mit denen zu jedem Zeitpunkt der Ort des Mondes bestimmt werden konnte. ${ }^{36}$ In letzter Konsequenz wird bei dieser Betrachtungsart der Mond auf einen hellen Punkt oder bestenfalls eine kleine Scheibe reduziert, an der man gerade noch die Phasen ablesen kann. Da es aber nicht um eine Kartografie des Mondes geht, ist diese Reduktion von Nutzen. Ein Beispiel für die Ermöglichung von Vorstellungen, die der Funktion der Analogie sehr nahe kommen, lautet so:

Unter die Beobachtungen mit dem Verkleinerungs-(ilas kann man auch die rechnen die Herr Deluc von dem (jletscher Büet aus gemacht hat. Die Rhone schien ihm nämlich in Vergleichung mit den ungeheuren Eisgebürgen nur ein kleiner Bach, und die Schätzung, daß diese Eisberge die Rhone Jahrhunderte durch unterhalten könnten, wurde dadurch erleichtert. ${ }^{37}$

Das Verkleinerungsglas besetzt in diesem Beispiel genau die Stelle, die das Mikroskop bzw. die Analogie bei Boyle innehatte: Es bringt eine Sache, die nicht recht fassbar war, in den Bereich der Vorstellbarkeit. Bereits dieses Beispiel betreibt eine Engführung zwischen dem ,praktischen Nutzen" des Verkleinerungsglases und dessen analogischer Überspitzung; diese wird an anderer Stelle noch deutlicher:

Wenn Scharfsinn ein Vergrößerungs-(ilas ist, so ist der Witz ein Verkleinerungs-Glas. Glaubt ihr denn daß sich bloß Entdeckungen mit Vergrößerungs-Gläsern machen ließen? Ich glaube mit Verkleinerungs-Gläsern, oder wenigstens durch ähnliche Instrumente in der Intellektual-Welt sind wohl mehr Entdeckungen gemacht worden. Der Mond sicht durch einen verkehrten Tubum aus wie die Venus mit bloßen $\Lambda$ uge und mit bloßen $\Lambda$ ugen wie die Venus durch einen guten Tubum in seiner rechten Lage. Durch ein gemeines Opern-Glas würden die Plejaden wie ein Nebelstern erscheinen. Die Welt, die so schön mit Bäumen und Kraut bewachsen ist, hält ein höheres Wesen als wir vielleicht eben deswegen für verschimmelt. Der schönste gestirnte Himmel sicht uns durch ein umgekehrtes Fernrohr leer aus. ${ }^{38}$

Diese letzte Bemerkung impliziert dic Steigerung und Übersteigung der Funktion des Hohlspiegels, mit dem die frühen Astronomen das gesamte Firmament in ihrem Blick hatten; durch die hier beschriebene massive

36 Lichtenberg: Schriften und Briefe $(\Lambda \mathrm{nm} .6)$, Kommentarband zu I und II, S. 168.

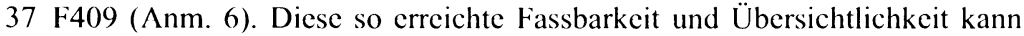
auch poetisch als die Übersichtlichkeit (,eusynopton') einer Handlung begriffen werden, vgl. Aristoteles: Poetik ( $\Lambda$ nm. 4), 1450b. 
Verkleinerung der Sterne verschwinden diese ganz. Das Beispiel von Mond und Venus lenkt den Blick freilich auf die Relativität von Größen bzw. die Ähnlichkeit von Mond und Venus hinsichtlich ihrer Phasen. Doch steht die Frage im Raum, welches Erkenntnispotenzial man sich von dem Verschwinden der Sterne versprechen kann. Blumenberg liest diese Passage - sowic die bereits erwähnten ${ }^{39}$ Fantasie-Vergrößerungen und -Verkleinerungen - als "Inbegriff der Beherzigungen des Traums“. Lichtenberg habe versucht, „das Ganze über den vielen Problemen der Beobachtung und des Experiments, die ihn ständig beschäftigten, nicht zu verlieren. ${ }^{40}$ Doch in dieser Passage liegt auch eine Warnung verborgen; diese ,Beherzigung des Traums“ offenbart und amplifiziert die massive Relativität jeglicher Wahrnehmung. Zwar ist der Witz mit seiner analogischen Verkleinerungsmöglichkeit im Stande, potenziell Alles auf Alles zu übertragen; aber tatsächlich, Alles` zu erblicken, führt zu Blindheit, falschen Einschätzungen, Katastrophen. Das Bild eines ,höheren Wesens' - das eben nicht, höher', sondern nur, größer` ist und mehr wahrnimmt - hat Lichtenberg in einem Anhang zu einem Aufsatz über den Walrat ausgeführt, wo das Traum-Ich Experimente an einer kleinen blaugrünen Murmel durchführt, die aber die Erde ist und durch das Experimentieren zerstört wird. ${ }^{41}$ Setzt man diesen Text zu dem , höheren Wesen ' in Verbindung, das unsere Welt für, verschimmelt' hält, so ist der Schluss Blumenbergs, dass mit den verschwundenen Sternen die „Leere im Universum genauer getroffen sei“42 - also ausschließlich ein Darstellungsproblem bewältigt wird -, zumindest zu relativieren; vielmehr ist die Totalanalogic, die alles auf einen Schlag sichtbar macht, eine Figuration der Apokalypse. Die vergleichende Rede der Analogie besetzt bei Lichtenberg mithin eine ubiquitäre Position, die sich ihrer generellen Unsicherheit nicht entledigen kann. Deshalb entwirft Lichtenberg verschiedene Argumentationen, diese rückzuversichern, z.B. über die Axiomatik der Moral oder die mathematisch-geometrische Evidenz qua dioptrischer Medien. Da dies aber allein übertragende Operationen sind, muss man mit der generellen Unsicherheit der generalisierten Analogie rechnen und/oder kann ihr Gegenteil mit den - nie zu unterschätzenden Mitteln der Stilkritik zum Schweigen bringen: „So ist zum Beispiel das Wort unvergleichlich im Deutschen ganz unvergleichlich erbärmlich. “4.3

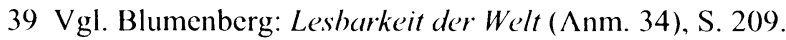

40 Blumenberg: Lesharkeit der Welt $(\Lambda \mathrm{nm} .34)$, S. 209, vgl. auch passim.

41 Vgl. Lichtenberg: Schriften und Briefe $(\Lambda \mathrm{nm} .6), \mathrm{Bd} .3, \mathrm{~S} .108 \mathrm{ff}$.

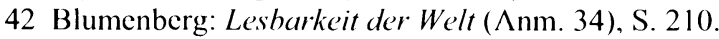

43 L141 (ヘnm. 6). 